\title{
A Composite Descriptor for Shape Image Retrieval
}

\author{
Xinjian Wang ${ }^{1, a}$, Guangchun Luo ${ }^{1, b}$ and Ke Qin ${ }^{1, c}$ \\ ${ }^{1}$ School of Computer Science and Engineering, University of Electronic Science and Technology of \\ China, Chengdu, 611731, China \\ awangxinjian_Iw@163.com, bgcluo@uestc.edu.cn, cqinke@uestc.edu.cn
}

Keywords: retrieval, special feature, shape matrix Fourier descriptor(SMFD), Histogram of Distances, Euclidean distance

\begin{abstract}
In order to quickly retrieval the interested image from the mass images, this paper proposed a composite descriptor based on contour shape and spacial feature for image retrieval. Firstly, we extract the contour by the shape matrix Fourier descriptor(SMFD).Then, use the adjacent contour sampling points and image center to form a triangle, the triangle have a only Circumcircle , use the radius of the Circumcircle to form a distance histograms, by adjusting the weight , combined the two methods, measures the similarity among the images with Euclidean distance. Finally, the experiment verified the feasibility and efficiency of the method. Experimental results show that the method is simple and fast, it has the character of transfer, rotation and scale invariant, it can achieve better retrieval accuracy and recall rates than the other method.
\end{abstract}

\section{Introduction}

With the rapid development of multimedia、Internet and computer vision technology, more and more digital images generated everyday. How to quickly and accurately obtain the desired image from the mass image data has become a hot research topic in the field of computer vision. At present, Content-based image has become the mainstream of image retrieval technology research and the focus in the domestic and foreign research.

Content-based image retrieval mainly includes the color-based image retrieval, texture-based image retrieval and shape-based image retrieval. Because when human visual identify objects, the most easy to distinguish according to the shape of the target object, so the shape features relative to the color and texture features are attracting more attention.

Based on the contour Shape retrieval, distance histogram、Boundary Moment[1]、Elastic Matching[2] 、Fourier Descriptors[3-12] 、Wavelet Descriptors[13-15] 、Curvature Scale Space [16-19] and Shape Contexts[20] are the typical method. But the above method only described the statistical characteristics of the contour points, ignore the contours of the spatial characteristics, which makes the retrieval results have a certain deviation with the physical application.

Therefore, we propose a new method of describing the contour shape. The method describe the statistical characteristic of contour based on shape matrix Fourier descriptor(SMFD), use the adjacent contour sampling points and image center to form a triangle, use the radius of the Circumcircle to describe the special characteristic. Combining the statistical distribution characteristic and the spatial characteristic can improve the performance of the shape image retrieval.

\section{Feature description}

Shape matrix Fourier descriptor. For example , Fig.1 is a pentacle, the target image uses the standard Canny operator to extract the target edge information, Suppose the centroid is $c\left(x_{c}, y_{c}\right)$, the longest distance of points on the contour to the centroid is R, C and R are defined as shown below: 


$$
\left\{\begin{array}{l}
x_{c}=\frac{1}{n} \sum_{i=0}^{n-1} x_{i} \\
y_{c}=\frac{1}{n} \sum_{i=0}^{n-1} y_{i} \\
R=\max \sqrt{\left(x_{i}-x_{c}\right)^{2}+\left(y_{i}-y_{c}\right)^{2}}
\end{array}\right.
$$

Where $x_{i}$ and $y_{i}$ are the coordinates of the equal interval sampling on target contour, $\mathrm{n}$ is the number of the equal interval sampling on target contour. The minimum circumscribed circle $\left(c\left(x_{c}, y_{c}\right)\right)$ is the center, $\mathrm{R}$ is the radius) of the object is used to be the object region, the establishment of a polar raster $(r-\theta)$ system based on the origin of the centroid of the object, contour interval sampling point distribution as statistical object, with the equal radius of concentric circles in radial direction( $\mathrm{dr}=\mathrm{R} / \mathrm{N}, \mathrm{N}$ is the number of equal radius) and equal circumference $(d \theta=360 / M, \mathrm{M}$ is the number of equal circumference) divide the minimum circumscribed circle, in order to establish the shape matrix with statistical meaning. The line of the shape matrix corresponds to the concentric rings, columns of the shape matrix corresponding to the rays, The elements of the matrix represent the number of contour points of the circular interval. Through the shape matrix production process, the shape matrix of statistics is the distribution of boundary points in polar coordinates of grating system, so, it has nothing to do with the starting points of image contour curve selection. In addition, the origin of polar coordinate system is taken at the centroid, can eliminate position translation . Considering the image possible occur scaling, matching the target image and the template image are used in the same equal way, namely takes the fixed $\mathrm{N}$ and $\mathrm{M}$ can meet the requirement of image scale invariance.

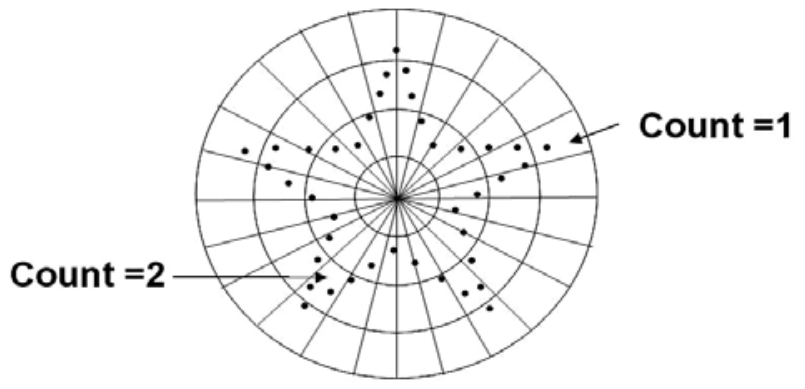

(a) Extract target edge

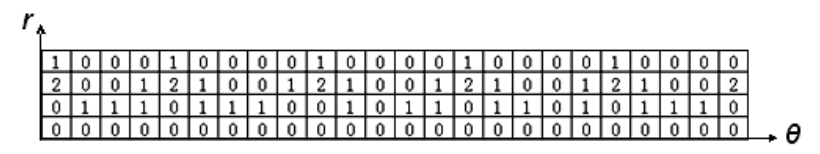

(b) Shape matrix

Fig.1: Schematic diagram of the shape matrix

Through the pretreatment process of the image can be seen that shape matrix satisfies the image features of the translation and scale invariant requirements, that is to say when the image translation and zoom, the shape matrix is unchanged, but the shape matrix are used to describe the target outline also exist certain problems, shape matrix does not fulfil the requirements of rotation invariance. In order to comparative and analysis, the conversion of the shape matrix into a one-dimensional vector, by comparing the original image shape matrix curve and its spin $d \theta$ 、 $5 d \theta$ 、11de, it will be not difficult to find, Rotating Image(An angle increment of $d \theta$ ) show the curve along the horizontal axis conducts periodic fluctuations(period $\mathrm{T}=\mathrm{M} * \mathrm{~N}$ ), reflected in the shape matrix is in the form of column to cycle along the axis shift, shape matrix is reflected in a column vector along the x-axis translation cycle. For cyclical curve, Fourier coefficients modulus value is approximately the same after Fourier transform, that is to say, Fourier transform was carried out on the curve shape matrix can fulfil the image rotation invariance.

For a periodic function $\mathrm{X}(\mathrm{t})$,its Fourier series can be expressed by Eq. 2.

$$
x(t)=\sum_{n=-\infty}^{\infty} a_{n} e^{j n \frac{2 \pi}{T} t}
$$


Its Fourier coefficients can be expressed by Eq. 3.

$$
a_{n}=\frac{1}{T} \int_{0}^{T} x(t) e^{-j n \frac{2 \pi}{T} t} d t
$$

Usually in front of a large number of $a_{n}$ to form a feature vector $u\left(\left|a_{0}\right|,\left|a_{1}\right|, \ldots,\left|a_{r}\right|\right)$ which is called Fourier descriptors. For closed curves, because the Fourier descriptor has good robustness ,so its widely used to description feature of the curve. This method is based on the shape matrix, expands it by column as a vector and performs Fourier transform, by constructing the shape matrix Fourier descriptor (SMFD) as the similarity measure between images.

Because the Fourier descriptor dimension is larger, if all the coefficients are included in the calculation of descriptors distance is easy to lead to results of noise on the curve of the sensitive; Fourier descriptor curves of different rotation angle is worth to broadly similar, Fourier descriptor curves of different rotation angle is similar, high frequency parts of the curve position remains unchanged, the amplitude is approximately the same, each curve in low frequency range have small change. Therefore, in order to make the similarity measure SMFD has good robustness, constructs the shape of a matrix Fourier descriptors by taking the part where the Fourier transform modulus is greater than the average value, its Fourier series can be expressed by Eq.4.

$$
S M F D=u\left(\left|a_{0}\right|,\left|a_{1}\right|, \ldots,\left|a_{\mathrm{r}}\right|\right), \quad\left|a_{\mathrm{i}}\right| \geq \frac{\sum_{\mathrm{j}=1}^{\mathrm{N}}\left|a_{\mathrm{j}}\right|}{\mathrm{N}}, \quad i \in(1, r)
$$

$\mathrm{N}$ is the number of Fourier transform coefficient of shape matrix curve, This value is typically a power of 2 . Assuming that the image similarity measure between I and $\mathrm{J}$ image with SMFD of Euclidean distance is $D 1_{I J}$, it can be expressed by Eq. 5 .

$$
D 1_{I J}=D\left(\mathrm{SFMD}_{1}, \mathrm{SFMD}_{2}\right)=\sqrt{\left(a_{0}^{I}-a_{0}^{J}\right)^{2}+\left(a_{1}^{I}-a_{1}^{J}\right)^{2}+\cdots+\left(a_{r}^{I}-a_{r}^{J}\right)^{2}}
$$

A shape description based on the Circumcircle. For boundary points after sampling, the centroid and the adjacent boundary points form a triangle, the triangle can uniquely identify a circumcircle. With the radius of circumcircle composed of a set of feature vector to describe the shape, the distribution of the radius value is established HD (Histogram of Distances).Fig.2 explain how to calculate the radius of a circle. In Fig.2, $\mathrm{C}$ is the shape of the centroid, A and $\mathrm{B}$ are two adjacent boundary sampling points. We suppose the edge of the triangle are $C A 、 C B$ and $A B$, the length of the three edges are L1, L2, L3, R can be calculated by the following formula:

$$
R=\frac{L 1 * L 2 * L 3}{\sqrt{(L 1+L 2+L 3) *(L 2+L 3-L 1) *(L 1+L 3-L 2) *(L 1+L 2-L 3)}}
$$

Fig.2: Circumscribed circle shape description

If the boundary point sampling is dense, L1 and L2 values are approximately equal, compare with L2 and L3, the value of L3 will be much smaller. In this case ,R is about half the length of the L1 and L2. Therefore, the boundary points do not densely sampling.

Assuming that the space similarity measure between the image I and J with HD Euclidean distance $D 2_{I J}$, it can be expressed by Eq.7. 


$$
D 2_{I J}=\mathrm{D}\left(H D_{1}, H D_{2}\right)=\sqrt{\sum_{K=1}^{N}\left(R_{I K}-R_{J K}\right)^{2}}
$$

Where, $\mathrm{N}$ is the dimension of the HD distribution histogram, where $R_{I K}, R_{J K}$ is the value of kdimension.

\section{Similarity measurement}

Use the $D_{I J}$ to measure the similarity between image I and image J,the $D_{I J}$ is defined as bleow.

$$
D_{I J}=\omega_{S F M D} D 1_{I J}+\omega_{H D} D 2_{I J}
$$

Where, $\omega_{\text {SFMD }}$ is the weight of shape matrix Fourier descriptor, $\omega_{H D}$ is the weight of Histogram of Distances based on circumcircle, $\omega_{\text {SFMD }}+\omega_{\text {HD }}=1$. The two features are used Euclidean distance to calculate the similarity, the Euclidean distance value is small, shows two images more similar, so the two image metric value D_IJ is smaller, the higher the similarity of the two image.

\section{The experimental results and analysis}

Evaluation index. The Composite method proposed in this paper named SFMD-HD, in order to prove the validity of the method, we select several typical shape recognition algorithm for experiments and compare with the method. Usually select the R (Recall rate) and P (Precision rate) to judge an image retrieval algorithm in the field of image retrieval, through these two indicators to quantitative analysis of the effect of the selected image retrieval algorithm.

$$
\mathrm{R}=\frac{r}{m}, \mathrm{P}=\frac{r}{n}
$$

Where, $\mathrm{r}$ is the images that meet the requirements in retrieval results. $\mathrm{m}$ is the total images that meet the requirements in the test data set $n$ is the total number of images.

MPEG-7 image set is used for performing experiments, this dataset includes 70 type of image, each type consists of 20 images, we select 10 type representative images from the image dataset, in practical application, the users pay more attention to the top search results, so we select the top 60 results to compare.

\section{Experimental results and analysis}

validation test. The method proposed in this paper, by adjusting the values of $\omega_{\mathrm{SFMD}}$ and $\omega_{\mathrm{HD}}$ in the set $\{0,0.2,0.4,0.6,0.8,1\}$,we can get different group. We present a number of experiments to verify our approach at different weight. Table 1 lists precision of all kinds of images.

Table 1: SFMD-HD retrieval performance under different weight

\begin{tabular}{ccccccc}
\hline$\omega_{\text {SFMD }}$ & 0 & 0.2 & 0.4 & 0.6 & 0.8 & 1 \\
\cline { 2 - 6 }$\omega_{H D}$ & 1 & 0.8 & 0.6 & 0.4 & 0.2 & 0 \\
\hline Apple & $58.6 \%$ & $58.9 \%$ & $60.6 \%$ & $62.3 \%$ & $61.9 \%$ & $59.7 \%$ \\
Bat & $47.1 \%$ & $47.5 \%$ & $47.6 \%$ & $50.2 \%$ & $48.8 \%$ & $47.3 \%$ \\
Bird & $43.5 \%$ & $43.6 \%$ & $44.8 \%$ & $49.1 \%$ & $45.4 \%$ & $43.7 \%$ \\
Butterfly & $47.8 \%$ & $48.3 \%$ & $48.7 \%$ & $49.3 \%$ & $48.1 \%$ & $48.0 \%$ \\
Camel & $40.2 \%$ & $42.1 \%$ & $43.3 \%$ & $45.7 \%$ & $45.2 \%$ & $43.6 \%$ \\
Cattle & $41.2 \%$ & $41.8 \%$ & $42.1 \%$ & $43.4 \%$ & $43.1 \%$ & $43.2 \%$ \\
Cup & $52.2 \%$ & $52.5 \%$ & $53.7 \%$ & $54.6 \%$ & $54.3 \%$ & $54.1 \%$ \\
Dog & $46.1 \%$ & $47.5 \%$ & $50.9 \%$ & $52.3 \%$ & $49.6 \%$ & $48.4 \%$ \\
Elephant & $51.1 \%$ & $51.4 \%$ & $52.6 \%$ & $53.1 \%$ & $52.8 \%$ & $51.7 \%$ \\
Hammer & $31.7 \%$ & $32.5 \%$ & $32.4 \%$ & $33.8 \%$ & $32.6 \%$ & $32.5 \%$ \\
\hline
\end{tabular}

We can see from the table 1 , when the $\omega_{\text {SFMD }}$ equal to 0.6 and $\omega_{\text {HD }}$ equal to 0.4 , the correct rate of image reaches the maximum, that means for grouping images, the contour information and spatial 
information Composite is necessary, $\omega_{\text {SFMD }}$ is greater than the $\omega_{H D}$ reflect human visual habits focuses on the main structure of the shape.

Performance verification. In order to evaluate the performance of our proposed SFMD-HD algorithm (denoted by M1) based on the shape contour feature, in the $\omega_{\text {sFм }}$ equal to 0.6 and $\omega_{\text {нд }}$ equal to 0.4 , compare our method with reference 21(denoted by M2) and reference 22(denoted by M3). M2 based on SC algorithm and dynamic programming algorithm is used to measure the distance between Shape Contexts distance, so, the computational efficiency is low. M3 based on contour complex coordinates Fourier transform, without considering spatial relationships between the contour shape. Fig. 3 shows the recall of the three methods, Fig. 4 shows the precision of the three methods. It can be seen that the proposed method is feasible and effectual.
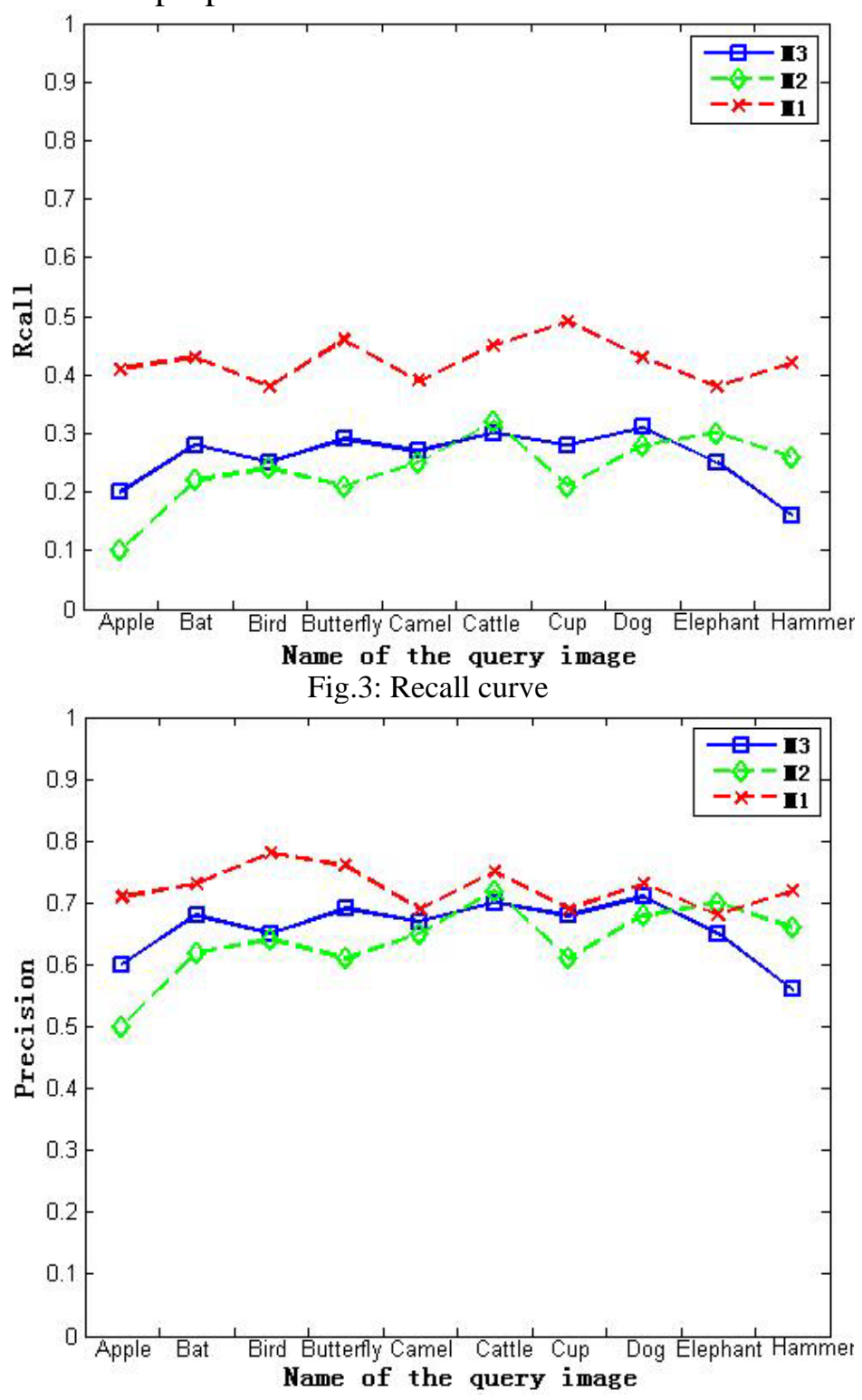

Fig.4: Precision curve

\section{Conclusions}

In this paper, we have proposed a new image retrieval fusion method, it pay attention to the contour shape feature and spatial feature. Experiments have been performed on the MPEG-7 images set., Experimental results prove that the proposed method is simple and practical,it can be used to deal with image retrieval problems. 


\section{Acknowledgements}

The research work was supported by Foundation of science \& technology department of Sichuan province under Grant No. 2012GZ0088 and No.2013JQ0005

\section{References}

[1] Chen CC. Improved Moment Invariants for Shape Discrimination [J]. Pattern Recognition, 26(5), pp.683-686, 1993.

[2] Bimbo AD,PalaP. Visual image retrieval by elastic matching of user sketches [J], IEEE Transactions on Pattern Analysis and Machine Intelligence, 19(2), pp.121-132, 1997.

[3] Akrem El ghazal, Otman Basir, Saeid Belkasim. Farthest point distance: A new shape signature for Fourier descriptors [J].Signal Processing: Image Communication, 24(7) ,pp.572-586,2009.

[4] Volodymyr V. KindraTenko. On Using Functions to Describe the Shape [J]. Journal of MathematicalImaging and Vision. 18(3),pp.225-245.2003.

[5] Emillio Musso, Lorenzo Nicolodi. Invariant Signatures of Closed Planar Curves [J].Journal of Mathematical Imaging and Vision.35(1),pp.68-85,2009.

[6] Granlund G H. Fourier preprocessing for hand print character recognition [J]. IEEE Transactions on Computers, 21(2),pp.195-201,1972.

[7] Persoon E, Fu K S. Shape discrimination using Fourier descriptors [J]. IEEE Transactions on Pattern Analysis and Machine Intelligence,8(3),pp.388-397. 1986.

[8] Oirrak A E, Daoudi M, Aboutajdin D. Affine invariant descriptors using Fourier series [J]. Pattern Recognition Letters,23(10),pp.1109-1118,2002.

[9] Bartolini I, Ciaccia P, Patella M. WARP:Accurate Retrieval of Shapes Using Phase of Fourier Descriptors and Time Warping Distance [J].IEEE Transactions on Pattern Analysis and Machine Intelligence, 27(1),pp.142-147,2005.

[10]Zhang D S, Lu G J. Study and evaluation of different Fourier methods for image retrieval [J], Image and Vision Computing,23(1),pp. 33-49,2005.

[11]Kunttu I, Lepisto L, Rauhamaa J, Visa A. Multiscale Fourier descriptors for defect image retrieval [J].Pattern Recognition Letters,27(2),pp.123-132, 2006.

[12]Zhang D S, Lu G J. A comparative study of curvature scale space and Fourier descriptors [J].Journal of Visual Communication and Image Representation,14(1),pp.41-60,2003.

[13] Gene C H, Chuang C C, Kuo J. Wavelet Descriptor of Planar Curves : Theory and Applications [J].IEEE Transactions on Image Processing, 5(1) ,pp. 56-70, 1996.

[14]Kong X D, Luo Q S, Zeng G H, et al. A new shape descriptor based on centroid radii model and wavelet transform [J], Optics Communications, 273,pp.362-366,2007.

[15] Yadav R B, Nishchal N K, Gupta A K, et al. Retrieval and classification of shape- based objects using Fourier, generic Fourier, and wavelet - Fourier descriptors technique : A comparative study

[16]Mokhtarian F, Bober M. Curvature Scale Space Representation: Theory Application and MPEG-7 Standardization[M], first ed., Kluwer Academic Publishers, Dordrecht, 2003.

[17]Abbasi S, Mokhtarian F, Kittler J. Curvature scale space image in shape similarity retrieval [J].Multimedia Systems, 7,pp.467-476, 1999.

[18]Abbasi S, Mokhtarian F, Kittler J. Enhancing CSS - based shape retrieval for objects with shallow concavities [J]. Image and Vision Computing, 18(3) ,pp.199-211, 2000.

[19] Mark S. Drew, Tim K. Lee, Andrew Rova. Shape retrieval with Eigen-CSS search [J]. Image and Vision Computing, 27(6),pp.748 -755, 2009.

[20]Belongie S, Malik J, Puzicha J.Shape matching and object recognition using shape contexts [J]. IEEE Transactions on Pattern Analysis and Machine Intelligence, 24(4) : pp.509-522, 2002.

[21]Xin Shu, Xiaojun Wu. A novel contour descriptor for 2D shape matching and itsapplication to image retrieval. Image and Vision Computing, 29(4):,pp.286-294, 2011.

[22]Zhang D S, Lu G J.Shape-based image retrieval using generic Fourier descriptor[J].Signal processing:Image,communication,17(10),pp.825-848, 2002. 